\title{
The diverse X-ray properties of four truly isolated elliptical galaxies: NGC 2954, NGC 6172, NGC 7052, and NGC 7785
}

\author{
E. Memola ${ }^{1, \star}$, G. Trinchieri ${ }^{1}$, A. Wolter ${ }^{1}$, P. Focardi ${ }^{2}$, and B. Kelm ${ }^{2}$ \\ 1 INAF-Istituto Nazionale di Astrofisica, Osservatorio Astronomico di Brera, via Brera 28, 20121 Milano, Italy \\ e-mail: elisabetta.memola@mib.infn.it \\ 2 Dipartimento di Astronomia, Università di Bologna, via Ranzani 1, 40127 Bologna, Italy
}

Received 13 August 2008 / Accepted 4 February 2009

\section{ABSTRACT}

\begin{abstract}
Aims. We investigate the X-ray properties of four isolated elliptical galaxies, selected from the Updated Zwicky Catalog according to strict isolation criteria. Isolated galaxies are not influenced by the group/cluster environment, and their X-ray emission can be studied independently of the often overwhelming contribution of the hot intergalactic medium. They are therefore suited to studying the X-ray characteristics relative to their intrinsic properties.

Methods. We analyzed our own XMM-Newton and archival Chandra data in detail for three objects, and derived, when possible, the spatial and spectral characteristics of each source. An upper limit for the fourth one was obtained from archival ASCA data. We compared their characteristics with those of other 23 isolated objects for which X-ray and optical data are available in the literature. We explored possible theoretical explanations to interpret our results.

Results. In spite of our attempt to select very homogeneous objects, both in terms of optical properties and environmental characteristics, we find a wide range in X-ray luminosities and $L_{X} / L_{B}$ ratios for the four objects: two of them show a hot gaseous halo, whereas no gas is detected in the other two, to a factor $>10$ in luminosity. In fact, we find a large spread in the $L_{X} / L_{B}$ for all galaxies considered, suggesting that the presence of hot gas is not easily related to the optical luminosity or to the mass, even in isolated systems. Younger objects tend to be less luminous in X-rays than older systems. However, it appears that older objects could span a wide range in luminosities.
\end{abstract}

Key words. X-rays: galaxies - galaxies: elliptical and lenticular, cD

\section{Introduction}

The high X-ray luminosity observed in early-type galaxies comes mostly from a diffuse hot component (e.g. Forman et al. 1985; Trinchieri \& Fabbiano 1985), while stellar sources dominate only at the low end of the X-ray luminosity distribution (e.g. Fabbiano \& Trinchieri 1985; Kim et al. 1992). It has long been known that the relation between the X-ray and the optical luminosity of early-type galaxies shows a large scatter (e.g. Canizares et al. 1987; Eskridge et al. 1995; Brown \& Bregman 1998; Beuing et al. 1999). The contribution from a group and its effects on the galaxy X-ray properties can explain some of this scatter (Helsdon et al. 2001). Massive ellipticals at the center of a group retain their own interstellar medium (ISM) and may also be embedded in the hot intragroup gas, which could enhance their observed X-ray luminosity (Canizares et al. 1983); at the same time, a galaxy moving at high speed through the intra-group medium may undergo ram-pressure stripping (Gunn \& Gott 1972) or viscous stripping (Nulsen 1982), thus decreasing its gaseous content without affecting its stellar luminosity much.

The majority of early-type galaxies are found in groups and clusters (Melnick \& Sargent 1977; Dressler 1980; Tully 1987), and dominant members are the most X-ray luminous examples of their type (Helsdon et al. 2001; O'Sullivan et al. 2001b). $\mathrm{X}$-ray selected samples are, thus, most likely biased towards

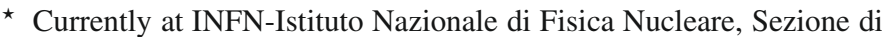
Milano Bicocca, Piazza della Scienza 3, 20126 Milano, Italy.
$\mathrm{X}$-ray bright, hence group dominant ellipticals. The influence of the environment has therefore been one of the major sources of confusion, since it is difficult to disentangle the group and the galaxy properties. On the other hand, it has already been noticed that the scatter in the $L_{\mathrm{X}}-L_{B}$ relation persists even when center group galaxies are not considered, and it is relatively large at any given mass. For instance, by excluding galaxies embedded in an intra-group medium (e.g. the EXG in Fukazawa et al. 2006), part of the scatter can be reduced, but only at high luminosities (i.e. most massive early-type galaxies). The careful selection of galaxies within an extremely poor environment is a step towards quantifying the environmental effects.

Many studies have addressed the issue of defining optical samples of isolated galaxies (e.g. Colbert et al. 2001; Smith et al. 2004; Reda et al. 2004; Denicoló et al. 2005) but unfortunately only a few have a follow-up in the X-ray band (e.g. O'Sullivan \& Ponman 2004; O'Sullivan et al. 2007). In this work we have considered a small, local sample selected with strict isolation criteria and studied its X-ray emission. We also intend to collect additional data (e.g. optical spectra to derive Lick indexes), to better define the global properties of our isolated ellipticals, which will help our understanding of their X-ray characteristics in the context of the current formation and evolution scenarios for ellipticals. While it is established that the stars in the most massive ellipticals were formed at high redshift (Ellis et al. 1997; Treu et al. 2002; Bernardi et al. 2003; van Dokkum 2005), much less is known about how massive ellipticals have been assembled (Andreon 2006; 
Table 1. Basic optical parameters for the isolated galaxies. Galaxy distances are Virgo-centric.

\begin{tabular}{|c|c|c|c|c|c|c|c|}
\hline Source & $\begin{array}{c}\text { Vel. } \\
\mathrm{km} \mathrm{s}^{-1}\end{array}$ & $\begin{array}{c}D \\
\mathrm{Mpc}\end{array}$ & $\begin{array}{l}\text { Scale } \\
\mathrm{kpc} /{ }^{\prime \prime}\end{array}$ & $\mathrm{Opt}_{\mathrm{dim}}$ & $\begin{array}{c}\log L_{B} \\
L_{B_{\odot}}\end{array}$ & $\begin{array}{c}\log L_{K} \\
L_{K_{\odot}}\end{array}$ & $\begin{array}{c}\log M_{*} \\
M_{\odot}\end{array}$ \\
\hline NGC 2954 & 3774 & 51 & 0.241 & $1.1 \times 1.7$ & 10.36 & 11.00 & 10.78 \\
\hline NGC 6172 & 5085 & 69 & 0.323 & $1.0 \times 1.0$ & 10.46 & 11.12 & 10.92 \\
\hline NGC 7052 & 5082 & 69 & 0.323 & $1.4 \times 2.5$ & 10.61 & 11.61 & 11.41 \\
\hline NGC 7785 & 3876 & 52 & 0.247 & $1.3 \times 2.5$ & 10.70 & 11.42 & 11.22 \\
\hline
\end{tabular}

De Propris et al. 2007; De Lucia et al. 2008), and current evidence has so far been unable to unambiguously prefer the monolithic scenario (e.g. Eggen et al. 1962; Larson 1975; Chiosi \& Carraro 2002) versus hierarchical merging (e.g. Toomre \& Toomre 1972; Knochfar \& Silk 2006). Moreover, in their study of a sample of isolated ellipticals, Marcum et al. (2004) find both good candidates for the monolithic assembly scenario and objects that bear clear signs of interaction (up to possible fossil groups). In the hierarchical merger scenario, mergers should occur at all times, and they might be observable in the field in the present epoch. Recent works studied the evolution of the Xray emission along the merging sequence (Nolan et al. 2004; Brassington et al. 2007), which can provide some prediction on the expected X-ray luminosity. In particular, post-merger ellipticals could be relatively devoid of the X-ray gas, possibly expelled at the nuclear merger stage, while older merger remnants could have had the time to rebuild a sizable X-ray halo (O’Sullivan et al. 2001a; Sansom et al. 2006). Observing isolated galaxies would allow us to study these effects independently of the environmental factor.

We discuss here the X-ray properties for the four early-type galaxies from the sample we selected (Section 2) for which $\mathrm{X}$-ray data exist. Throughout this paper we assume $H_{0}=$ $75 \mathrm{~km} \mathrm{~s}^{-1} \mathrm{Mpc}^{-1}$. We normalize optical $B$-band and $K$-band luminosities to the $B$-band and $K$-band luminosity of the sun, $L_{B_{\odot}} \sim 5 \times 10^{32} \mathrm{erg} \mathrm{s}^{-1}$, and $L_{K_{\odot}} \sim 5.67 \times 10^{31} \mathrm{erg} \mathrm{s}^{-1}$ respectively.

\section{The sample of isolated ellipticals}

The four elliptical galaxies we discuss in this paper are part of a larger sample of bright isolated galaxies (Focardi \& Kelm 2009), which we selected from the Updated Zwicky Catalog (UZC, Falco et al. 1999) applying an adapted version of the Focardi \& Kelm (2002) neighbour search code. The latter is a versatile tool that can be applied to $3 \mathrm{D}$ galaxy catalogs to select galaxy samples characterized by different values of the luminosity and/or belonging to different environments (from the extreme field to galaxy clusters). UZC is a wide angle 3D catalog of nearby galaxies that covers the northern sky down to a declination $\delta \sim-2.5^{\circ}$, and is claimed to be $96 \%$ complete for galaxies brighter than $m_{B}=15.5$. The code was already successfully applied to UZC and to the 2dFGRS (Colless et al. 2001; Colless et al. 2003) to extract different homogeneous samples (see Focardi \& Kelm 2002; Kelm et al. 2005; Focardi et al. 2006; Focardi \& Kelm 2009).

To select the sample of isolated galaxies four basic criteria were applied to the UZC: a) minimum $B$ luminosity $\left(L_{B}=1.3 \times\right.$ $\left.10^{10} h_{75}^{-2} L_{B_{\odot}}\right)$; b) velocity range $\left.\left(v_{r} \in[2500-5000] \mathrm{km} \mathrm{s}^{-1}\right) ; \mathbf{c}\right)$ galactic latitude $\left(\left|b^{\mathrm{II}}\right| \geq 15^{\circ}\right)$; and $\left.\mathbf{d}\right)$ no companion galaxies in $3 \mathrm{D}$ space (within a given radius $R_{\mathrm{iso}}=1.3 h_{75}^{-1} \mathrm{Mpc}$ and radial velocity $\left|\Delta v_{r}\right|=1000 \mathrm{~km} \mathrm{~s}^{-1}$ ).

The lower limit in optical luminosity increases the chance of detecting X-ray emission; in fact, we are interested in checking the presence of an extended, hot, X-ray emitting ISM, and no detectable extended halo is expected from optically faint galaxies (Trinchieri \& Fabbiano 1985; Canizares et al. 1987; O'Sullivan et al. 2001b). The completeness limit of the catalog implies a search for companions with $L_{B} \geq 5.5 \times 10^{9} h_{75}^{-2} L_{B \odot}$ (from $m_{B}=15.5$ at the upper limit of the radial velocity range adopted, $\left.v_{\mathrm{r}}=5000 \mathrm{~km} \mathrm{~s}^{-1}\right)$. The lower limit in radial velocity was imposed to reduce distance uncertainties due to peculiar motions and any contamination by the Virgo cluster, while the galactic latitude restriction avoids an artificial increase of isolated galaxies at low latitudes due to galactic extinction.

Isolated ellipticals must be selected in an objective way and their isolation must be proved on a scale comparable to the one of galaxy groups and clusters and to some depth (i.e. considering neighbours within a wide magnitude range). Isolation is granted in our sample on the typical cluster/group scale for companions down to $\sim 2$ mag fainter and having velocity difference well above the typical velocity dispersion in clusters.

As a whole the sample contains 43 galaxies, among which 8 are classified as early-type galaxies. For only four of these X-ray data exist: NGC 2954 and NGC 7785, for which we obtained XMM-Newton data, NGC 7052, available in the Chandra archive, and NGC 6172 available in the ASCA archive. We list in Table 1 the basic optical data for the four early-type galaxies discussed here ${ }^{1}$. The Virgo-corrected recession velocities and $m_{B_{\mathrm{TC}}}$ used to derive the $B$-band luminosities are from LEDA; the optical dimensions and 2MASS $K$-band magnitudes and extinction used to calculate the $K$-band luminosity come from NED (Schlegel et al. 1998); the stellar mass is estimated from the $K$-band luminosity following the relation of Bell et al. (2003) and using the $B-V$ colors from LEDA.

Since the original selection of the sample, new data have become available that provide magnitudes (mostly $K$-band) and a few redshifts for potential companion galaxies. We checked that, with the new data, potential companions are still at least $\sim 2$ mag fainter than our sample galaxies, confirming our original selection. We further notice that NGC 2954 and NGC 7785 are present in the AMIGA database (Verdes-Montenegro et al. 2005, http: //amiga.iaa.es: 8080/DATABASE/) as CIG 358 and CIG 1045, and are also included in the Smith et al. (2004) sample. NGC 6172 is listed as isolated in Colbert et al. (2001) and Reda et al. (2004).

\section{X-ray observations}

\subsection{XMM-Newton data}

We obtained XMM-Newton observations for NGC 2954 and NGC 7785 between June and November 2004, with exposure times of $\sim 30 \mathrm{ks}$ (see Table 2 for observation

\footnotetext{
1 Note that the recession velocity values in Table 1 are Virgo-corrected and for this reason exceed, in two cases (NGC 6172 and NGC 7052), the original limit imposed on UZC heliocentric radial velocities.
} 
Table 2. Observation log.

\begin{tabular}{lcccccc}
\hline \hline Source & Obs. Date & $\mathrm{ks}^{*}$ & $\mathrm{cts} / \mathrm{s}(0.5-5.0 \mathrm{keV})$ & $r_{\mathrm{ext}}$ & Filter & Instrument \\
\hline NGC 2954 & 20041103 & $29.4 / 30.3 / 19.5$ & $(1.42 \pm 0.37 / 1.58 \pm 0.36 / 6.21 \pm 0.87) \times 10^{-3}$ & $30^{\prime \prime}$ & Medium & MOS1/MOS2/pn \\
NGC 7785 & 20040614 & $22.0 / 23.0 / 14.6$ & $(4.73 \pm 0.25 / 4.59 \pm 0.23 / 19.7 \pm 0.53) \times 10^{-2}$ & $120^{\prime \prime}$ & Thin & MOS1/MOS2/pn \\
NGC 7052 & 20020921 & 9.63 & $(7.38 \pm 0.31) \times 10^{-2}$ & $50^{\prime \prime}$ & - & ACIS-S \\
NGC 6172 & 19990215 & $57.2 / 52.1$ & - & $90^{\prime \prime}$ & - & GIS/SIS \\
\hline
\end{tabular}

* Live time of the central CCD after cleaning from high background flaring events (see text).

details). The EPIC detectors operated in PrimeFullWindow mode. We reprocessed the data with the XMM-Newton Science Analysis Software (XMM-SAS) version 6.5.0, using the latest calibration products. To analyze the scientific data we also used SAOImage DS9, FTOOLS, CIAO version 3.4, and Funtools.

We cleaned the XMM-Newton data from high flaring background periods using the standard technique described in the User's Guide to the XMM-Newton Science Analysis System. We first extracted a high-energy (10-15 keV) light-curve with time-bin $=100 \mathrm{~s}$ and excluded all "flaring" intervals using the standard count-rate cut (RATE $=0.35 \mathrm{cts} / \mathrm{s}$ for EPIC-MOS and RATE $=1.0 \mathrm{cts} / \mathrm{s}$ for EPIC-pn). Taking into account the Good Time Intervals (GTIs) and selecting single and double events (PATTERN $\leq 4)$ for the pn, and single, double, triple, and quadruple events (PATTERN $\leq 12$ ) for the MOS, we constructed the clean event files. We created X-ray images in different energy bands and with different pixel-binning for the three EPIC instruments. We also produced mask files that correspond to bad pixels, bad columns and CCD gaps, which were used to exclude them from the analysis. For the morphological studies we also merged the EPIC-MOS1 and EPIC-MOS2 event files. Otherwise, we used the instrument files separately to obtain the spectral photon distribution of our targets (see Sect. 4.3).

\subsection{Archival data}

We searched the X-ray archives for additional observations of the isolated galaxies in the sample. We found Chandra data for NGC 7052 and ASCA data for NGC 6172. NGC 7052 was observed with the Chandra ACIS-S instrument in September 2002. The instrument operated in faint mode, and observed the target for about $10 \mathrm{ks}$. Upon checking the data quality, we found that the event files provided were not contaminated by high background or flaring intervals, and could therefore be used to create images and extract spectral data (CIAO version 3.4), in analogy to what we did for the XMM-Newton data.

NGC 6172 was observed with ASCA on February, 1999 for $57.2 \mathrm{ks}$. We analyzed the data following the guidelines described in "The ASCA Reduction Guide" found at http://heasarc. gsfc.nasa.gov/docs/asca/abc/abc.html. We used a 90" radius source region, corresponding to $50 \%$ of the encircled energy fraction for the ASCA PSF, and a wider nearby circle for estimating the background. We derived a $\sim 1.4 \sigma$ positive signal (not sufficient to claim a detection of the source), which corresponds to a flux $F_{\text {tot(0.5-2.0) }} \sim$ a few $10^{-15} \mathrm{erg} \mathrm{cm}^{-2} \mathrm{~s}^{-1}$ (see Table 2). At the distance of the source, this corresponds to a luminosity of $L_{\mathrm{tot}(0.5-2.0)} \sim 1 \times 10^{40} \mathrm{erg} \mathrm{s}^{-1}$, a value that we will use to estimate the maximum total luminosity of this object.

\section{X-ray spatial and spectral analysis}

\subsection{X-ray maps}

For both XMM-Newton and Chandra data-sets we produced $\mathrm{X}$-ray images with $2^{\prime \prime} \times 2^{\prime \prime}$ pixel resolution in the energy bands $0.5-2.0 \mathrm{keV}, 2.0-4.5 \mathrm{keV}$, and $4.5-7.5 \mathrm{keV}$. We adaptively smoothed all of them with the csmooth tool, from CIAO, with a minimum $\mathrm{S} / \mathrm{N}$ of 3. X-ray contours from the smoothed images in the $0.5-2.0 \mathrm{keV}$ band, which maximizes the $\mathrm{S} / \mathrm{N}$ for all sources, are plotted over the DSS optical images in Figs. 1-3 (left panels).

NGC 2954 and NGC 7785 show relevant emission only in the $0.5-2.0 \mathrm{keV}$ band, whereas NGC 7052 is visible both in the $0.5-2.0 \mathrm{keV}$ and in the 2.0-4.5 keV images. NGC 2954 and NGC 7052 are associated with compact and azimuthally symmetric sources, centered on the optical galaxy and of similar extent. NGC 7785 shows a more complex morphology, with the $\mathrm{X}$-ray contours clearly elongated in East-West direction. Two compact sources and an additional enhancement at $\geq 7^{\prime}$ SouthEast are also visible in the image. Faint optical counterparts appear associated with the two compact sources while the more diffuse peak to the South-East coincides with the cluster of galaxies $\mathrm{ZwCl} 2353.2+0535$. It is likely therefore that the extension is due to the presence of interlopers, rather than to emission related to the galaxy itself. We will examine more quantitatively this evidence in the next section.

\subsection{Radial surface brightness profiles}

To determine the extent of the emission and the possible presence of a nucleus, we examined the radial profiles in both XMM-Newton and Chandra data-sets. We centered them on the peak of the X-ray emission and calculated the radial surface brightness from the azimuthally averaged distribution of concentric annuli, having excluded all CCD gaps/defects. The maximum radius of the source is where the total profile changes slope and becomes consistent with the expected X-ray profile of the background. We used a circular region outside this radius, but close to the source, to estimate the background. The net profiles (cts/arsec ${ }^{2}$ ) in the $0.5-2.0 \mathrm{keV}$ band are shown in Figs. 1-3 (right panels), together with a comparison with the instrumental Point Spread Function (PSF). We parametrized the EPIC PSF with a King-type function: $\operatorname{PSF}(r)=A\left(1+\left(r / r_{\mathrm{c}}\right)^{2}\right)^{\alpha}$, where $A$ is the normalization, $r_{\mathrm{c}} \sim 5^{\prime \prime}-66^{\prime \prime} 6$ is the core radius (MOS and pn respectively) and $\alpha \sim-1.5$ is the slope (see the XMM-SOCCAL-TN-0022 and XMM-SOC-CAL-TN-0029 documents online at http : / / xmm.vilspa. esa.es). For the PSF of Chandra we used the mkpsf tool of CIAO with the spectrum appropriate to the source (see next section).

The profile of NGC 2954 is consistent with the XMM-Newton PSF, and shows a positive signal only out to $30^{\prime \prime}$. 

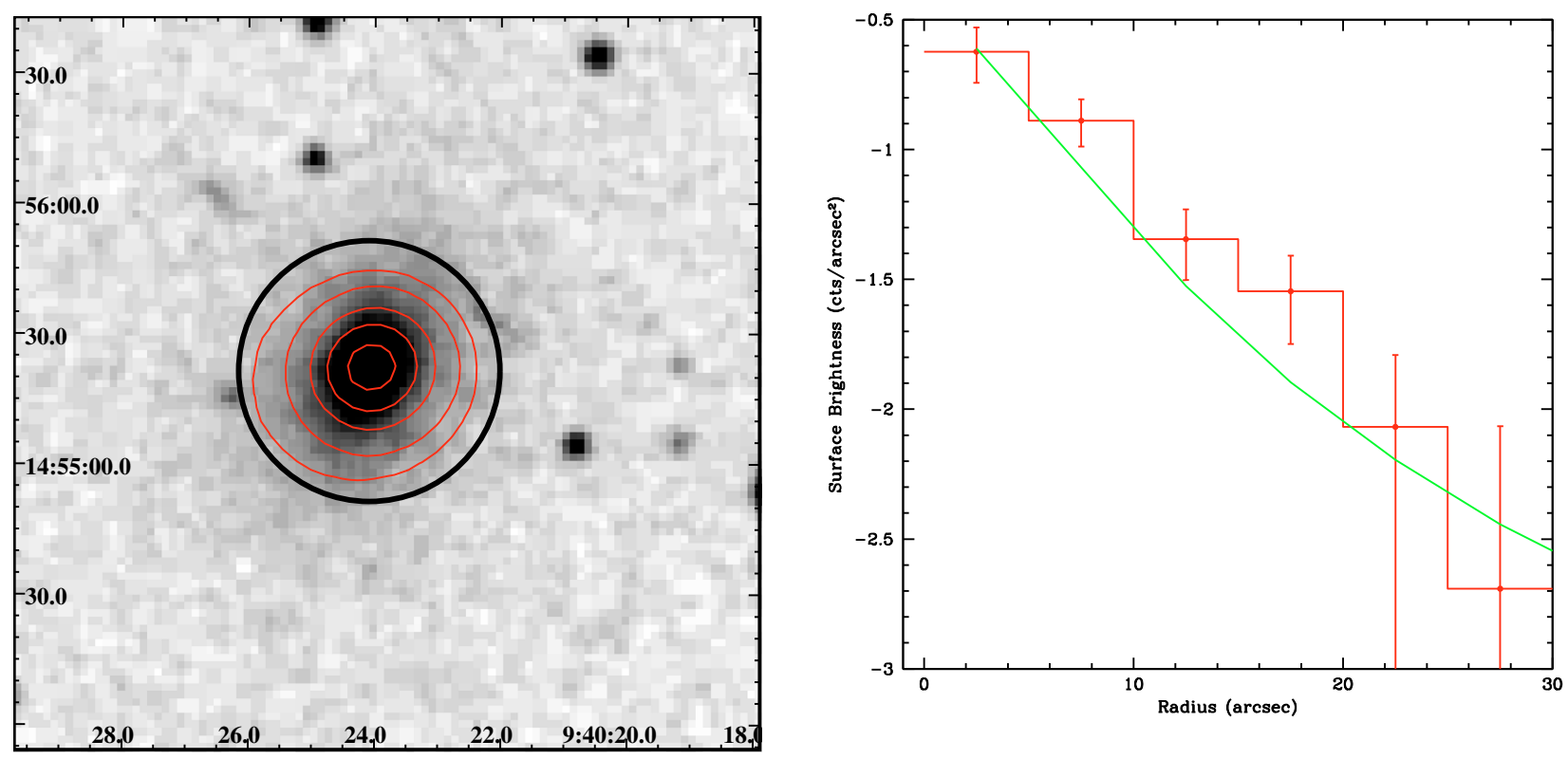

Fig. 1. Left panel: X-ray contours from the smoothed EPIC-MOS image (0.5-2.0 keV band) superposed onto the Digitized Sky Survey image (from SAO-DSS) for NGC 2954. A circle of 30" radius, corresponding to the X-ray extraction radius used for the spectral analysis, is shown. Right panel: Histogram: net radial surface brightness profile centered at the X-ray peak from the EPIC-pn data in the $0.5-2.0 \mathrm{keV}$ energy band. Continuous line: XMM-Newton Point Spread Function (see text).
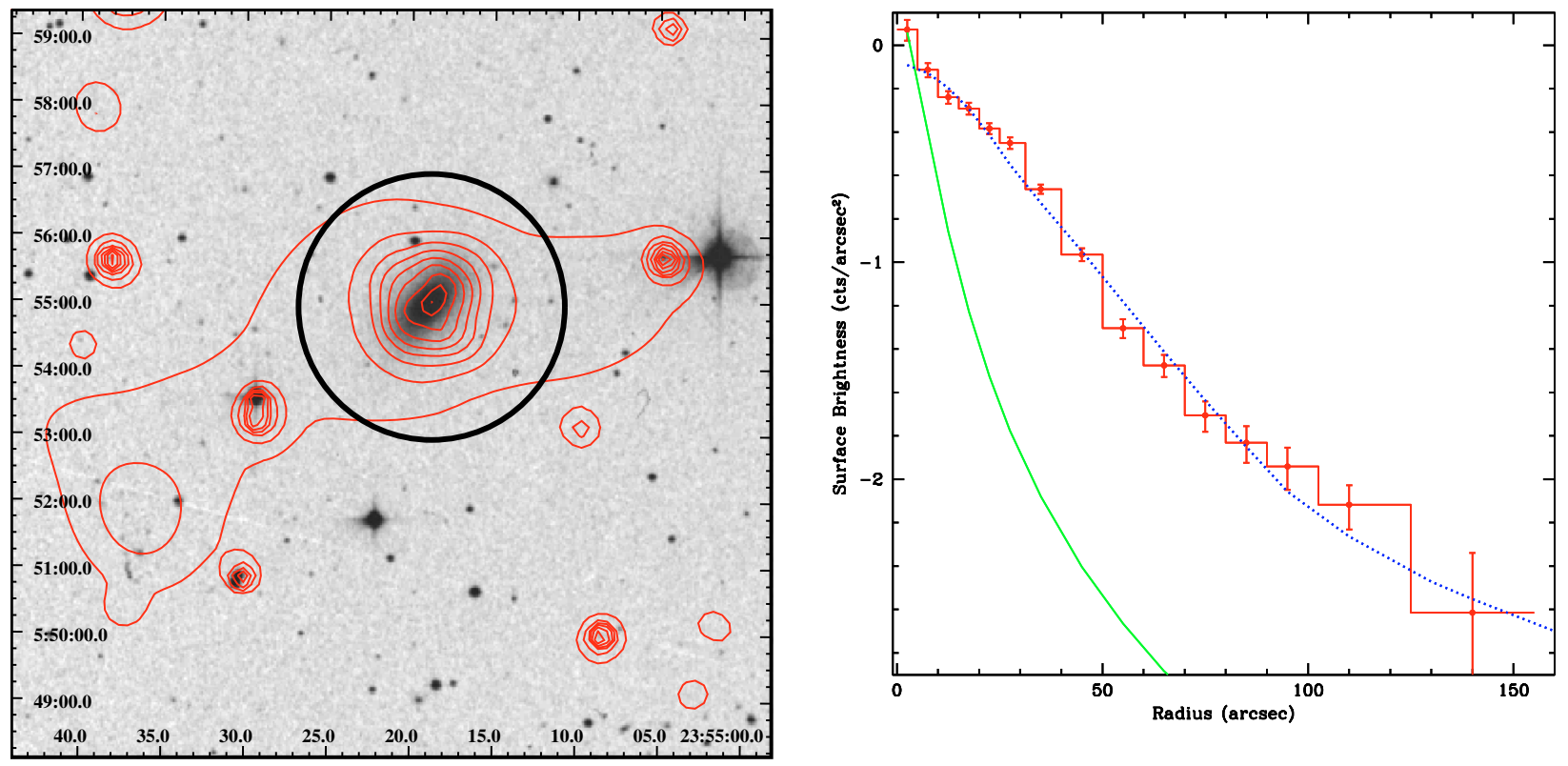

Fig. 2. Same as Fig. 1 for NGC 7785. The extraction radius shown in the left panel corresponds to $120^{\prime \prime}$. The dotted line represents the best fit $\beta$-model for NGC 7785 (see text for details).

Given the complex morphology of NGC 7785 described above, we analyzed the radial profile of the emission in four different azimuthal sectors, delimited by $90^{\circ}, 170^{\circ}, 240^{\circ}$, and $315^{\circ}$ counterclockwise from North. We excluded the visible compact sources with a $25^{\prime \prime}$ radius circle. Figure 4 shows the raw surface brightness profiles in the angular sectors defined above. We used EPIC-MOS images, because they are less affected by CCD gap regions. We confirm that no significant asymmetry in the emission is present from different directions and that the emission does not extend beyond $\sim 120^{\prime \prime} \div 160^{\prime \prime}$. The peak visible at $\sim 280^{\prime \prime} \div 380^{\prime \prime}$ in the SE region is most likely associated with extended emission from the $\mathrm{ZwCl} 2353.2+0535$ cluster not properly subtracted. We then parametrized the azimuthally averaged net profile with a King-type model, convolved with the XMM-Newton PSF described above. We find that a model with $r_{0} \sim 35^{\prime \prime}$ and $\beta \sim 0.95$ provides a good fit down to the central regions, consistent with no significant central excess, which could be the signature of emission from a nuclear source. The derived $\beta$-model is plotted in Fig. 2 (right panel).

The net profile of NGC 7052 presented in Fig. 3 (right panel) is consistent with the broad band profile previously presented and parametrized by Donato et al. (2004), as part of their sample 

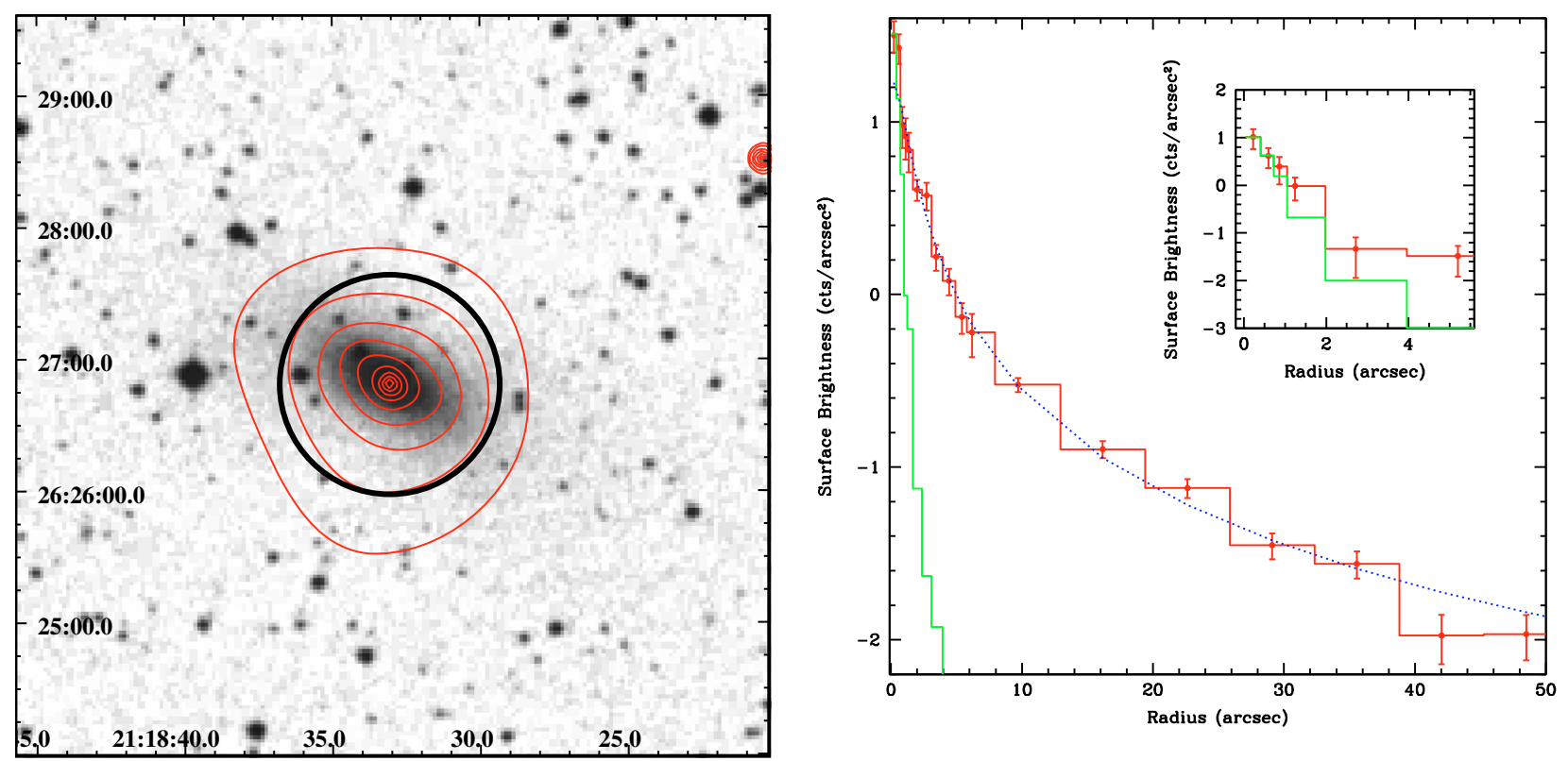

Fig. 3. Left panel: X-ray contours from the smoothed Chandra-ACIS-S image (0.5-2.0 keV) superposed onto the Digitized Sky Survey image (from SAO-DSS) for NGC 7052. A circle of 50"radius, corresponding to the X-ray extraction radius used for the spectral analysis, is shown. Right panel: Histogram (red): net radial surface brightness profile centered at the X-ray peak from ACIS-S data in the $0.5-2.0 \mathrm{keV}$ energy band. The Chandra simulated Point Spread Function is also represented with an histogram (green). The inset in the right panel shows a zoom of the net radial surface brightness profile in the 2.0-5.0 keV energy band, and the corresponding PSF (see color figure online). The dotted line represents the best fit $\beta$-model for NGC 7052 (see text for details).

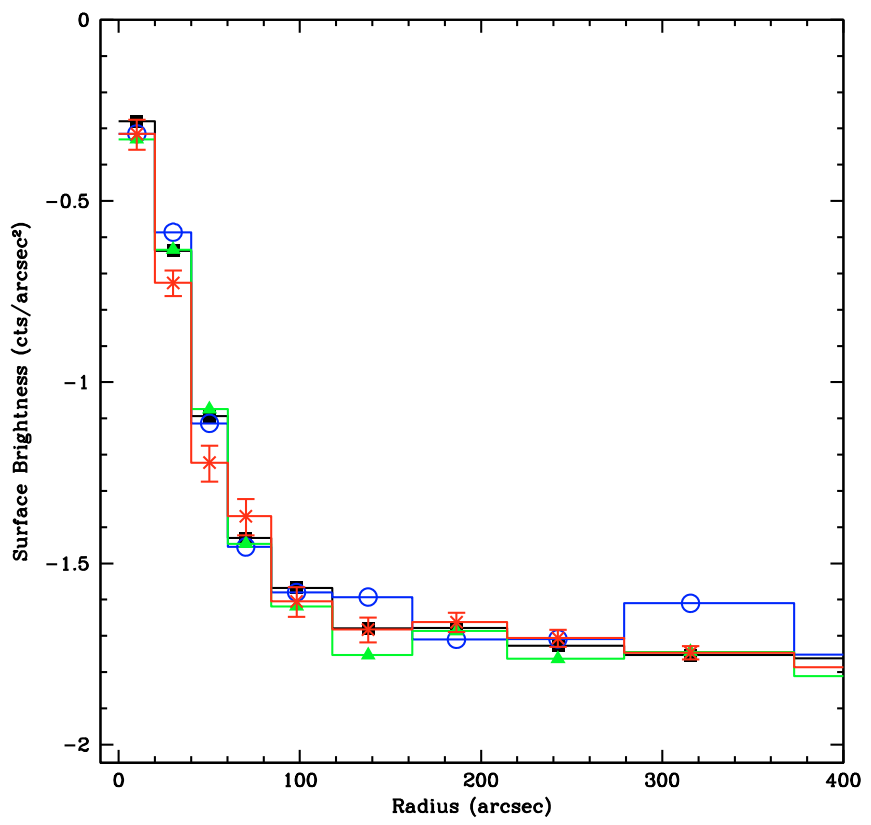

Fig. 4. EPIC-MOS raw surface brightness profiles (with no background subtraction) of NGC 7785 in the angular sectors defined in the text (see Sect. 4.2): 1) N (black line and squares); 2) SE (blue line and open circles); 3) SW (green line and triangles); 4) W (red line and crosses). For clarity, we only report the error-bars relative to the Western profile (see color figure online).

of FRI galaxies (NGC 7052 is the radio source B2 2116+26). Their best fit parametrization with a $\beta$-model $\left(r_{\mathrm{c}} \sim 1.11^{\prime \prime}\right.$, $\beta \sim 0.48)$ superposed on the data in the right panel of Fig. 3 indicates a small central excess, consistent with their result suggesting the presence of a point source at the $95.6 \%$ confidence. With our different choice of energy intervals, we are able to notice that the central point source becomes evident at energies $E>2 \mathrm{keV}$, while there is only a marginal excess over the best-fit $\beta$-model in the $0.5-2.0 \mathrm{keV}$ band. The inset in Fig. 3 shows a blow up of the inner part of the $2.0-5.0 \mathrm{keV}$ profile, where the photon distribution is entirely consistent with the Chandra PSF. As shown by the $0.5-2.0 \mathrm{keV}$ profile in Fig. 3, the total emission in NGC 7052 extends out to $50^{\prime \prime}$.

\subsection{X-ray spectra}

We extracted EPIC source counts of all three instruments separately from a circular region, centered on the source, with a radius of $30^{\prime \prime}$ for NGC 2954 and of $120^{\prime \prime}$ for NGC 7785 . Background spectra were extracted from circular source-free regions of $50^{\prime \prime}$ and $\sim 90^{\prime \prime}$ respectively, close to the target. The ancillary response matrix (ARF) and the detector response matrix (RMF) were created by means of the XMM-SAS tasks arfgen and rmfgen. The Chandra source and background spectra for NGC 7052, together with the appropriate spectral matrixes, were extracted using specextract from two circular regions with a radius of $50^{\prime \prime}$ each.

The X-ray spectra were analyzed with the XSPEC package (version 11.3.1; Arnaud 1996). EPIC-MOS1, -MOS2 and -pn spectra were fitted simultaneously. The source counts were grouped into energy bins such that each bin contains more than 20 counts to use the chi-square $\left(\chi^{2}\right)$ statistics and has a significance level of at least $2 \sigma$ after background subtraction. The quoted errors on the best-fit parameters correspond to the $90 \%$ confidence level for one interesting parameter (i.e., $\Delta \chi^{2}=2.71$; Avni 1976). We used a power law to model the $\mathrm{X}$-ray binaries or the AGN component (usually parametrized 
Table 3. Summary of the spectral results. The EPIC-pn/ACIS-S net counts are derived in a circle of radius $r_{\text {ext }}$, given in the third column.

\begin{tabular}{|c|c|c|c|c|c|c|c|c|c|c|}
\hline Source & $\begin{array}{l}N_{\mathrm{H}_{\mathrm{gal}}} \\
\mathrm{cm}^{-2}\end{array}$ & $\begin{array}{c}r_{\mathrm{ext}} \\
\prime \prime\end{array}$ & $\begin{array}{c}\text { cts } \\
\text { pn/ACIS-S }\end{array}$ & $\begin{array}{c}k T_{\text {mekal }} \\
\mathrm{keV}\end{array}$ & $\Gamma$ & $\begin{array}{l}N_{\mathrm{H}_{\text {int }}} \\
\mathrm{cm}^{-2}\end{array}$ & $\chi^{2} /$ d.o.f. & $\begin{array}{c}L_{\text {mekal }(0.5-2.0)} \\
\mathrm{erg} \mathrm{s}^{-1}\end{array}$ & $\begin{array}{c}L_{\mathrm{po}(2.0-10)} \\
\mathrm{erg} \mathrm{s}^{-1}\end{array}$ & $\begin{array}{l}L_{\mathrm{LMXBs}}^{* * *} \\
\mathrm{erg} \mathrm{s}^{-1}\end{array}$ \\
\hline NGC 2954 & $3.43 \times 10^{20}$ & 30 & $132 \pm 19$ & - & $1.9 \pm 0.3$ & - & $10.36 / 12$ & - & $3.2 \times 10^{39}$ & $1.1 \times 10^{40}$ \\
\hline NGC 2954 & $3.43 \times 10^{20}$ & 30 & $132 \pm 19$ & 0.54 & $1.9^{*}$ & - & $8.10 / 11$ & $5.4 \times 10^{38 * *}$ & $2.5 \times 10^{39}$ & $1.1 \times 10^{40}$ \\
\hline NGC 7052 & $13.6 \times 10^{20}$ & 50 & $719 \pm 30$ & $0.48 \pm 0.06$ & $1.4_{-0.8}^{+0.4}$ & & $47 / 48$ & $1.4 \times 10^{41}$ & $5.3 \times 10^{40}$ & $4.5 \times 10^{40}$ \\
\hline NGC 7052 & $13.6 \times 10^{20}$ & 50 & $719 \pm 30$ & $0.48 \pm 0.06$ & $1.9^{*}$ & $9_{-0.7}^{+1.1} \times 10^{21}$ & $46 / 48$ & $1 \times 10^{41}$ & $5 \times 10^{40}$ & $4.5 \times 10^{40}$ \\
\hline NGC 7785 & $5.24 \times 10^{20}$ & 120 & $3340 \pm 87$ & $0.56 \pm 0.02$ & $2.0 \pm 0.3$ & - & $263 / 254$ & $8 \times 10^{40}$ & $2.0 \times 10^{40}$ & $3.0 \times 10^{40}$ \\
\hline
\end{tabular}

* Fixed; ${ }^{* *}$ note that this is not a statistically significant component. We use it to derive an upper limit of $L_{\mathrm{X}} \leq 1.5 \times 10^{39} \mathrm{erg} \mathrm{s}^{-1}$ for the plasma component (see text for details); ${ }^{* * *} L_{(2.0-10)}$ expected from LMXBs, calculated using the relation in Kim \& Fabbiano (2004), taking into account the appropriate $K$-band luminosity and energy range.

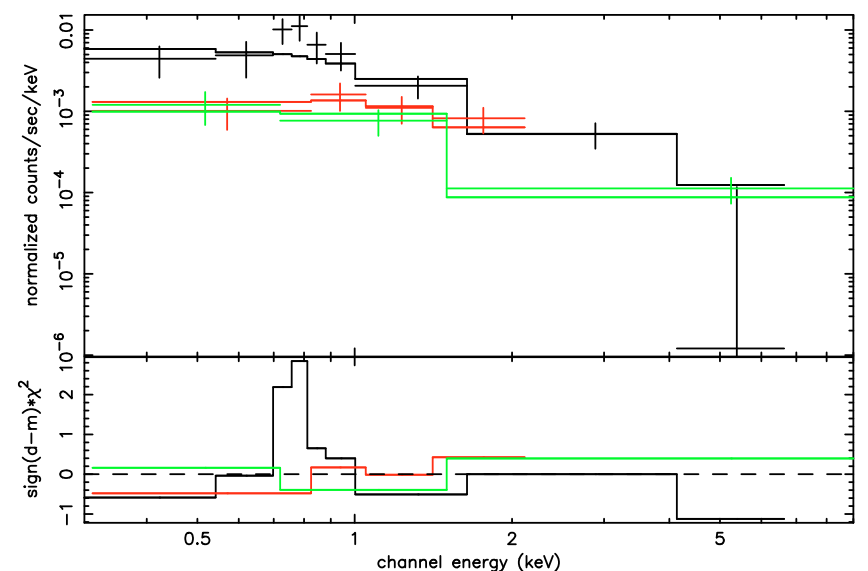

Fig. 5. NGC 2954: XMM-Newton EPIC-MOS1 (red), -MOS2 (green), and -pn (black) spectrum and $\chi^{2}$ behavior. The best-fit model is a power law (see color figure online).

by $\Gamma=1.7 \div 1.9$ ), and a mekal component, with abundances fixed at $50 \%$ the solar value, to fit the diffuse hot gas. For both components, the appropriate Galactic Hydrogen column density $\left(N_{\mathrm{H}}\right)$ along the line of sight (Dickey \& Lockman 1990) has been taken into account. The spectral fitting results are summarized in Table 3.

\subsubsection{NGC 2954}

A simple power law with a photon index $\Gamma=1.9$ is a good fit and the X-ray luminosity is $L_{\mathrm{po}(2.0-10)}=3.2 \times 10^{39} \mathrm{erg} \mathrm{s}^{-1}$. However, the residuals (see Fig. 5) are not uniformly distributed, and show an excess at $\sim 0.8 \mathrm{keV}$. If we parametrize this with a mekal model, keeping the power law fixed at the best fit $\Gamma=1.9$, we derive a gas temperature of $0.54 \mathrm{keV}$ for a luminosity $L_{\text {mekal }(0.5-2.0)}=5.4 \times 10^{38} \mathrm{erg} \mathrm{s}^{-1}$. The additional mekal component is not required by the statistics, but it allows us to derive an upper limit on the gas contribution. We estimated a limit with a $>95 \%$ confidence by raising the mekal component normalization until $\Delta \chi^{2}>4$ (see Avni 1976). With this normalization the upper limit value is $L_{\text {mekal }(0.5-2.0)}=1.5 \times 10^{39} \mathrm{erg} \mathrm{s}^{-1}$.

\subsubsection{NGC 7052}

Two components are required to fit the spectral data: a mekal component with a temperature of $0.5 \mathrm{keV}$, and a power law with photon index value $\Gamma=1.4$. The power-law slope appears to be flatter than expected from a population of binaries.
Moreover, the evidence of a central point source in the radial profile (see also Donato et al. 2004) suggests that we could expect a contribution from a central AGN. In AGN, slopes as flat as $\Gamma=1.4$ have been reported, however they have often been interpreted as the result of a more typical power-law spectrum (i.e. $\Gamma=1.9$ ) heavily suppressed at low energy by large absorption and poorly sampled at high energies, due to limited statistics and energy band (e.g. Nandra \& Pounds 1995; Caccianiga et al. 2004; Piconcelli et al. 2005). Therefore, we derive an alternative spectral parametrization assuming a power law with index fixed at $\Gamma=1.9$, which gives an intrinsic absorption $N_{\mathrm{H}}=9_{-0.7}^{+1.1} \times 10^{21} \mathrm{~cm}^{-2}$ (see Table 3). This gives an unabsorbed luminosity $L_{\mathrm{po}(2.0-10)}=5 \times 10^{40} \mathrm{erg} \mathrm{s}^{-1}$. With either model for the hard energy emission, the plasma component has $L_{\text {mekal }(0.5-2.0)} \sim 1 \times 10^{41} \mathrm{erg} \mathrm{s}^{-1}$. Both models are equally plausible and give the same luminosity in the hard component. Moreover, the plasma component has also the same luminosity of $\sim 10^{41} \mathrm{erg} \mathrm{s}^{-1}$ in both cases.

In order to better define the contribution of the AGN component separately from the one of the unresolved X-ray binaries we also extracted the X-ray spectrum of NGC 7052 in two separate, but contiguous, regions: a $3^{\prime \prime}$ radius circle and a concentric annulus, with $3^{\prime \prime}$ and $50^{\prime \prime}$ radii. In the inner region, for a power law with index fixed at $\Gamma=1.9$, the $N_{\mathrm{H}}$ value is consistent, within the errors, with the value in Table 3 . This component has $L_{\mathrm{po}_{(2-10)}}=2.8 \times 10^{40} \mathrm{erg} \mathrm{s}^{-1}$, which we consider due to the AGN, since the binary emission should be small due to the area considered. Given the low statistics of the data at high energies, we cannot easily constrain the model, therefore we assume a power law with fixed slope $(\Gamma=1.9)$ and $N_{\mathrm{H}}$ at the galactic value to describe the binary emission in the outer annulus, which results in an unabsorbed luminosity $L_{\mathrm{po}_{(2-10)}}=2.2 \times 10^{40} \mathrm{erg} \mathrm{s}^{-1}$.

As already mentioned in the previous section, NGC 7052 is part of the sample of FRI galaxies discussed by Donato et al. (2004). We are in substantial agreement with these authors. However, given our different aims, considering the whole source out to a radius of $50^{\prime \prime}$, rather than to $1.5^{\prime \prime}$ as done by Donato et al. (2004), has provided us with significantly more photons, allowing a better definition of the different spectral components and a handle on the modelling of the high energy excess. In fact, the request of a power-law to model the high energy part of the spectrum is significant in our data, as shown by Fig. 6 (Left panel). The difference in the total luminosity $L_{(0.3-8)}=4 \times 10^{40} \mathrm{erg} \mathrm{s}^{-1}$ calculated by Donato et al. (2004), with respect to our $L_{(0.5-10)}=15 \times 10^{40} \mathrm{erg} \mathrm{s}^{-1}$ is entirely due to the wider aperture we use, which includes the contribution of the "whole" galactic component (plasma and the X-ray binaries). The estimate of the AGN luminosity is slightly higher than 

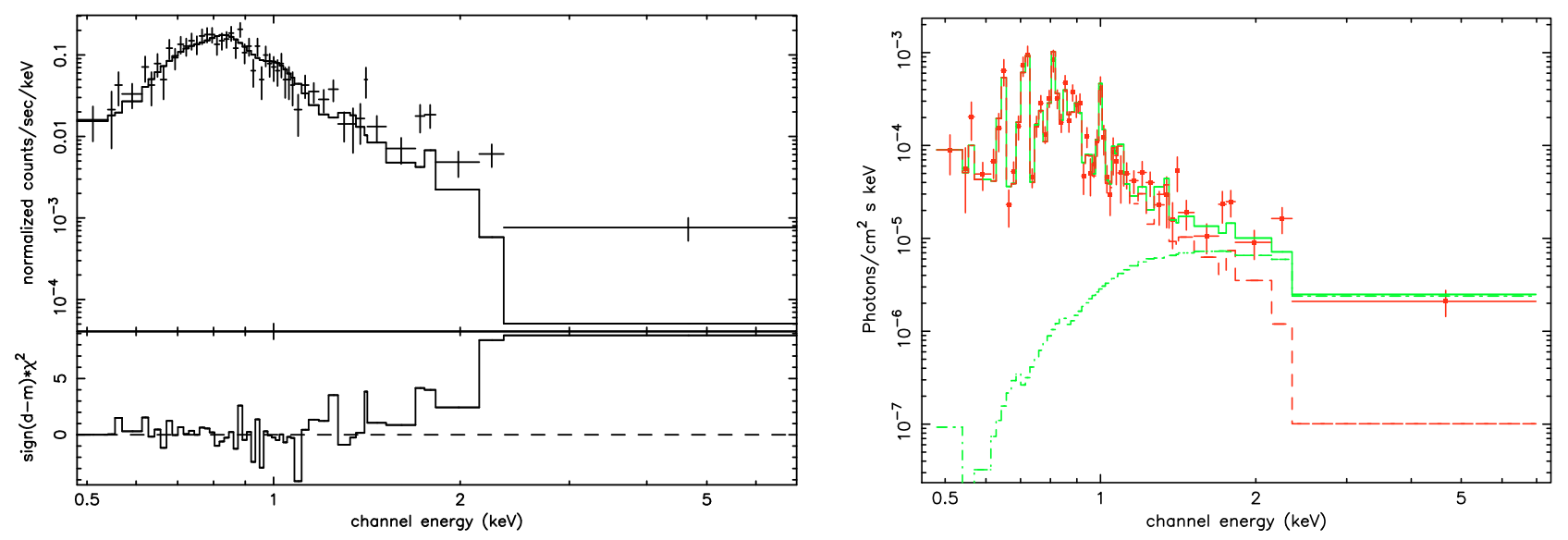

Fig. 6. Left panel: Chandra ACIS-S X-ray spectrum and $\chi^{2}$ behavior for NGC 7052, fitted with a single mekal component. Right panel: unfolded ACIS-S spectrum, with the best fit model composed of an absorbed power law plus a thermal component.
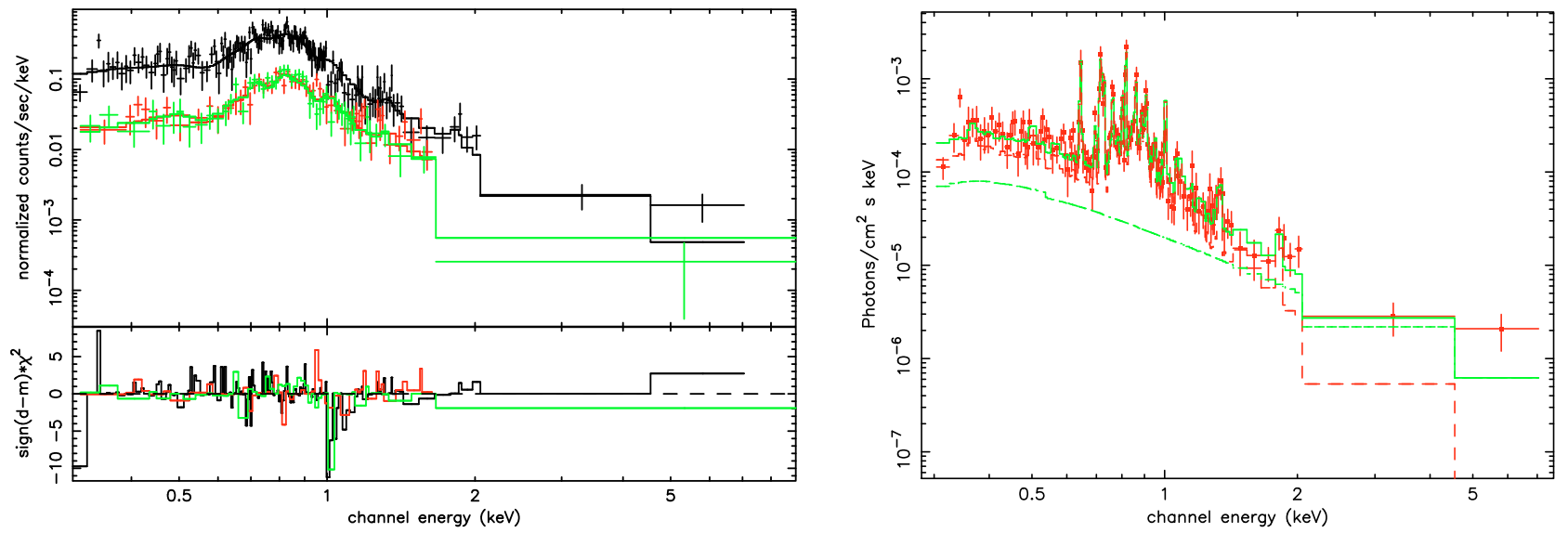

Fig. 7. Left panel: XMM-Newton EPIC-MOS1 (red), -MOS2 (green), and -pn (black) spectrum and $\chi^{2}$ behavior for NGC 7785. Right panel: unfolded X-ray spectrum derived from the EPIC-pn data. The best-fit model is a power law plus a thermal component (see color figure online).

the upper limit given in Donato et al. (2004). This could be due to different spectral assumptions, which they do not explicitly report.

\subsubsection{NGC 7785}

The spectrum requires both a power-law and a mekal component with $\Gamma=2.0$ and $\mathrm{kT}=0.56 \mathrm{keV}$, respectively (see Fig. 7 and Table 3). Both components are well determined: the plasma component dominates at low energies $(<2 \mathrm{keV})$, with an intrinsic $L_{\text {mekal(0.5-2.0) }}=8 \times 10^{40} \mathrm{erg} \mathrm{s}^{-1}$ to be compared with $L_{\mathrm{po}(0.5-2.0)}=1.4 \times 10^{40} \mathrm{erg} \mathrm{s}^{-1}$. At higher energies $(>2 \mathrm{keV})$, there is the sole contribution of the power-law, with $L_{\mathrm{po}(2.0-10)}=$ $2.0 \times 10^{40} \mathrm{erg} \mathrm{s}^{-1}$.

\section{X-ray emission from isolated galaxies}

In spite of the homogeneous selection criteria and environmental characteristics (see Sect. 2) our 4 isolated elliptical galaxies display different X-ray properties (see Sect. 4, Table 3). The total luminosities differ considerably, from $L_{X}>10^{41} \mathrm{erg} \mathrm{s}^{-1}$ for NGC 7785 and NGC 7052 to $L_{X} \sim 3 \times 10^{39} \mathrm{erg} \mathrm{s}^{-1}$ for NGC 2954 $(0.5-10 \mathrm{keV})$. No overall detection is obtained for NGC 6172 resulting in a total luminosity $L_{\mathrm{X}}<10^{40} \mathrm{erg} \mathrm{s}^{-1}$.

NGC 2954 has a compact morphology consistent with the instrumental PSF, and its spectrum is well represented by a power-law component. Given the lack of evidence of a nuclear source, we attribute this emission to the binary population. The contribution from binary sources is also detected in NGC 7052 and NGC 7785, with a luminosity of $L_{(2.0-10)} \sim 2 \times 10^{40} \mathrm{erg} \mathrm{s}^{-1}$. In all three cases this contribution is consistent with what is observed in other galaxies, based on their $L_{X}$ vs. $L_{K}$ ratio (see, e.g. Kim \& Fabbiano 2004), as indicated by the estimated luminosities reported in the last column of Table 3 .

The nucleus of NGC 7052 is resolved in the radial profile and has a luminosity of $\sim 3 \times 10^{40} \mathrm{erg} \mathrm{s}^{-1}$ (see also Donato et al. 2004). We found no evidence of a nuclear source in the X-ray data of NGC 7785, either in the profile or in the spectral properties. Evidence of an active nucleus is provided by radio emission (Condon et al. 2002), and its classification as a core galaxy by Lauer et al. (2007), with a $\sim 8.5 M_{\odot}$ central black hole (van der Marel \& van den Bosch 1998). However, the $1.4 \mathrm{GHz}$ radio power of $\log L_{1.4}=22.07 \mathrm{~W} \mathrm{~Hz}^{-1}$ indicates that the nucleus is relatively faint (it is also about an order of magnitude fainter than NGC 7052, at $\log L_{1.4}=23.13 \mathrm{~W} \mathrm{~Hz}^{-1}$; Condon et al. 2002), and could very well be below the sensitivity of current X-ray data.

We detect a hot ISM in NGC 7052 and NGC 7785, with an extent of 16 and $30 \mathrm{kpc}$ in radius, respectively, $L_{\mathrm{X}} \sim 10^{41} \mathrm{erg} \mathrm{s}^{-1}$ $(0.5-2.0 \mathrm{keV})$ and a temperature $\mathrm{kT} \sim 0.5 \mathrm{keV}$. We have also estimated a maximum contribution from a hot thin plasma component in NGC 2954, $L_{X} \leq 1.5 \times 10^{39} \mathrm{erg} \mathrm{s}^{-1}$ (Sect. 4.3.1), which is significantly lower than in the other two objects. Moreover, 
if we compare it with the X-ray luminosity expected from the integrated coronal emission from the stellar component (as estimated for example from the bulge of M 31, M32, the Galactic Ridge, and NGC 821, Bogdán \& Gilfanov 2008; Revnivtsev et al. 2007, 2006; Sazonov et al. 2006; Pellegrini et al. 2007), we expect that the hot gas contribution is really negligible in this galaxy.

From the spectral parameters of the hot plasma in NGC 7052 and NGC 7785, we estimate a gas mass $M_{\text {gas }} \sim 2.2 \times 10^{9} M_{\odot}$ for NGC 7052 and $\sim 4.6 \times 10^{9} M_{\odot}$ for NGC 7785 , assuming a volume corresponding to the maximum observed radius and spherical symmetry. These mass values are consistent with the present day stellar mass-loss rate from asymptotic giant branch stars of $\dot{M}_{*}=0.078\left(L_{B} / 10^{10} L_{B_{\odot}}\right) M_{\odot} \mathrm{yr}^{-1}$ (Athey et al. 2002) integrated over the age of the galaxies.

Using the evidence of a hot halo, we can also estimate the total mass of these two galaxies. To calculate the total mass we have used the expression in Fabricant et al. (1984), derived under the assumption that the gas is isothermal in hydrostatic equilibrium in the potential, worked out explicitly in terms of $\beta$ and $R_{\text {core }}$ by Mulchaey \& Zabludoff (1999). Applying their Eq. (3) to NGC 7052 and NGC 7785 with the appropriate parameters, we derive $M_{\text {tot }} \sim 5 \times 10^{11} M_{\odot}$ out to a radius of $16 \mathrm{kpc}$ for NGC $7052\left(\mathrm{M} / \mathrm{L}=12.3 M_{\odot} / L_{B_{\odot}}\right)$ and $M_{\text {tot }} \sim 1.9 \times 10^{12} M_{\odot}$ out to a radius of $30 \mathrm{kpc}$ for NGC $7785\left(\mathrm{M} / \mathrm{L}=37.9 M_{\odot} / L_{B_{\odot}}\right)$.

Comparing them with the estimates of the visible mass (see Table 1), these values indicate a large dark matter halo only in NGC 7785 (about an order of magnitude larger than the stellar mass). While we have no support from the data that the assumption of hydrostatic equilibrium is correct, and therefore the total masses derived from the X-ray data could be incorrect, nevertheless we note that NGC 7785 has a large M/L ratio derived independently of the stellar velocity dispersion (Heckman 1983). We cannot derive the total mass for the other two objects from the X-ray data. However, Heckman (1983) gives a mass for NGC 2954 of $M \sim 5 \times 10^{11} M_{\odot}$ within the effective radius (normalized to our $H_{0}$ value), which also suggests a large amount of dark matter in this galaxy.

To better estimate the diversity of the X-ray properties of the isolated galaxies, in particular in their ISM content, we compare them in Fig. 8, where we plot the $L_{X}$ of the gas vs. $L_{B}$. In the narrow range of optical luminosity, to within a factor of 3 , the X-ray luminosity of the ISM spans more than 2 orders of magnitudes, in particular if we consider that the upper limit to the "plasma" luminosity estimated for NGC 2954 could in fact be significantly lower due to a significant contribution expected from the stellar component. As discussed above, the total mass does not appear to be a better tracer of a hot ISM, even based on just the estimates for NGC 2954 and NGC 7785.

To better populate the region, and to exclude that the scatter is due to the very small sample used, we have searched the literature for isolated early-type galaxies for which a measure of the gas component is available. We plot 23 additional objects in Fig. 8. The galaxies are selected with isolation criteria similar to ours, from the sample of O'Sullivan et al. (2001b, 2007: ESO107-4, NGC 57, IC1531, and NGC 7796), and Helsdon et al. (2001: NGC 6776 and NGC 2271, plus seven galaxies with a somewhat less stringent isolation radius of $0.4 \mathrm{Mpc}$, mostly upper limits), plus NGC 2865 (Fukazawa et al. 2006), and NGC 821 (Pellegrini et al. 2007). We also include four "fossil" groups (NGC 6482 and ESO306-17: O'Sullivan et al. 2001b (note that the total mass of NGC 6482 could be significantly lower than typical fossil groups, see Buote et al. 2007); NGC 1132: Mulchaey \& Zabludoff 1999; and

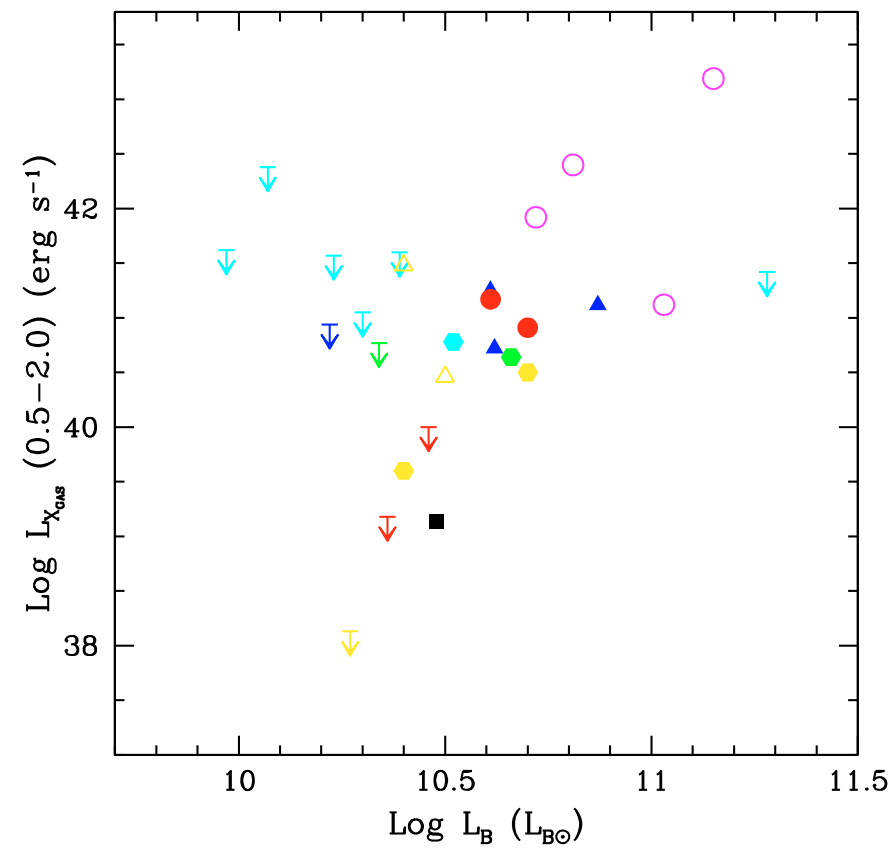

Fig. 8. Scatter plot of the $0.5-2.0 \mathrm{keV}$ hot gas X-ray luminosity vs. $B$-band luminosity of 23 early-type isolated galaxies and 4 isolated early-type pairs (see color figure online). $L_{\mathrm{X}}$ refer to the gas component contribution in $0.5-2.0 \mathrm{keV}$ except the 4 sources plotted as hexagons, for which only the total $L_{\mathrm{X}}$ is available. Arrows indicate upper limits. Red dots and arrows: this paper; blue triangles: O'Sullivan et al. (2007); blue arrow: O'Sullivan et al. (2001b); green hexagon and arrow: Helsdon et al. (2001) with no companion within $1.0 \mathrm{Mpc}$; cyan hexagon and arrows: Helsdon et al. (2001) with no companion within $0.4 \mathrm{Mpc}$; magenta open circles: "fossil" group ellipticals from O'Sullivan et al. (2001b), and Mulchaey \& Zabludoff (1999), and "fossil" cluster with data from Fukazawa et al. (2006); black square: Fukazawa et al. (2006); yellow hexagons and open triangles: Grützbauch et al. (2007); yellow arrow: Pellegrini et al. (2007).

NGC 1600: Fukazawa et al. 2006), which would also pass our isolation criteria, and 4 isolated early-type pairs (Grützbauch et al. 2007).

The distribution of galaxies in the plot of Fig. 8 indicates that the two bands cover very different luminosity ranges and there is a notable lack of optically bright and X-ray faint objects. The first is nothing but a different representation of what has been known now for a few decades, namely that the hot gas properties appear to be relatively independent of the optical ones. We merely use a well defined sample of isolated galaxies, therefore supporting the evidence that this (lack of) relation is intrinsic to this class of sources and not induced by environmental effects. We need instead to better understand whether the second effect is due to a selection bias. The sample we use in this work is made of galaxies of relatively low luminosity. This could be related to an evidence of a general lack of luminous isolated earlytype galaxies in the local universe (e.g. Kelm \& Focardi 2004; Sulentic et al. 2006), but it could also be a consequence of the small volume probed. In fact a work by Stocke et al. (2004) indicates that galaxies forming in poorly populated environments are just as likely to become very luminous elliptical galaxies as those in dense groups and in rich clusters. We note that, as shown by Fig. 8 itself, a small relaxing of the search parameters in the volume of the universe probed (in recession velocity or declination, rather than in the isolation criteria) has already provided us with a few brighter objects. 
The galaxies plotted in Fig. 8 do not represent a complete sample, therefore the lack of objects in the lower right corner can be simply due to the fact that we have not included any. There is no observational restriction to populate it, since current instruments have the sensitivity to detect X-ray luminosities below the $\sim 10^{40} \mathrm{erg} \mathrm{s}^{-1}$ limit in a very large volume. However, neither the large sample used by O'Sullivan et al. (2001b; 401 earlytype galaxies with ROSAT data selected regardless of environments, and possibly biased towards X-ray bright objects), nor the low X-ray luminosity objects, selected for this specific property (O'Sullivan \& Ponman 2004; David et al. 2006), appear to populate this region.

While not conclusive, this evidence suggests that bright galaxies in general do retain their hot ISM. We can further speculate that an optical luminosity $\log L_{B} \sim 10.5 L_{\mathrm{B}_{\odot}}$ could be used as a discriminant between two groups, with galaxies below such value unable to retain a hot halo, which instead, when present, is at the level of $10^{40} \mathrm{erg} \mathrm{s}^{-1}$ or above in optically brighter objects. A possible counterexample is given by the low-luminosity ( $L_{B} \leq 3 \times 10^{10} L_{\mathrm{B}_{\odot}}$ ) early-type galaxies from David et al. (2006), in which hot gas is detected in most of the 18 galaxies investigated at $L_{\mathrm{X}}(0.5-2.0 \mathrm{keV})=2 \times 10^{38}-2 \times 10^{40} \mathrm{erg} \mathrm{s}^{-1}$. However, the sample includes objects in different environments, which could modify their intrinsic properties (in fact the brightest object, NGC 4552, is in Virgo and it is currently being stripped of its gas content, Machacek et al. 2006), and lower luminosity objects appear to be in a wind phase, which would therefore limit any gas accumulation (David et al. 2006). Moreover, at the lower X-ray luminosity, the exact contribution from hot gas might be difficult to assess with data of limited quality, due to both the contribution from stellar sources and the possible presence of outflowing gas. This is exemplified by NGC 3379, a very well studied nearby elliptical galaxy with deep Chandra observations (Brassington et al. 2008): as discussed by Revnivtsev et al. (2008), most of the soft diffuse emission is well explained by the stellar population. This is also the only galaxy in which gas in outflow has been detected, but its contribution is very low, at $L_{\mathrm{gas}} \sim 4 \times 10^{37} \mathrm{erg} \mathrm{s}^{-1}, M_{\mathrm{gas}} \sim(3 \pm 1) \times 10^{5} M_{\odot}$ (Trinchieri et al. 2008b), well below previous estimates for a gas component. It is to be hoped that more detailed studies of these objects will become available, to help us understand better whether all isolated galaxies at low optical luminosities contain as little gas as speculated here.

\section{Comparison with theoretical expectations}

Theoretical models developed by Ciotti et al. (1991) and Pellegrini \& Ciotti (1998) discuss different yields in the X-ray band as a function of several input parameters, linked to the stellar and total mass and their distribution, and the $\mathrm{SN}$ Ia rate. Two specific examples are given for systems with $L_{B}=5 \times 10^{10} L_{\mathrm{B}_{\odot}}$ and $M_{*}=1.9 \times 10^{11} M_{\odot}$, and $L_{B}=10^{11} L_{\mathrm{B}}$ and $M_{*} \stackrel{\odot}{=}$ $4.2 \times 10^{11} M_{\odot}\left(H_{0}=50 \mathrm{~km} \mathrm{~s}^{-1} \mathrm{Mpc}^{-1}\right)$. In the former example the galaxies are able to develop partial to global winds that make the X-ray luminosity in the gas component drop to very low values. At larger masses, even in the partial wind regime, the galaxies would have a wider central inflow region due to the deeper potential well, with resulting higher gas temperatures and $L_{X}$ in the range $3 \times 10^{40} \mathrm{erg} \mathrm{s}^{-1}$ to $2 \times 10^{41} \mathrm{erg} \mathrm{s}^{-1}$. These values roughly correspond to our crude evaluation of two regimes discussed above. In this very simple scenario, NGC 7052 and NGC 7785 would be able to retain their hot ISM to levels consistent with the integrated stellar mass loss (see previous section), while the lack of gas in NGC 2954 and NGC 6172 could be explained as being due to a global wind that has cleaned them of the gas shed by the stars. Global winds are hard to detect since they are expected to produce very low X-ray luminosities, beyond the sensitivity and quality of our observations, as already discussed in the previous section.

Similarly, David et al. (2006) suggest that supernova heating is enough to drive a galactic wind in systems with $L_{K}<10^{11}$ $L_{K_{\odot}}$, even without resorting to the energy input from a central AGN. Consistently, they find that the low luminosity objects they analyze are also relatively under-luminous in their gaseous component, with gas masses below the expected accumulation from the stellar evolution. In spite of the general trend of having low $\mathrm{X}$-ray luminosities, their sample spans a wide range (about a factor $\sim 100$ ) in the $L_{X} / L_{K}$ ratios. David et al. (2006) interpret this as possibly due to the influence of AGN heating and/or environment (which is usually a major concern in most discussions). Since we have selected galaxies to be isolated, the wide range in luminosity ratios we find should not result from the role played by the environment.

The role of AGN feedback is often invoked to explain many galaxy properties, like for instance the tight scaling relations between Super Massive Black Hole masses and the properties of host galaxies' bulges (e.g. Ferrarese \& Merrit 2000; Marconi \& Hunt 2003), but the details of the mechanisms are not well know yet. Cavities, bubbles and weak shocks generated by the jet in the ISM demonstrate the ability of the AGN to deposit large amounts of energy in the environment (most evident in clusters, but now visible in galaxies as well, e.g. Finoguenov \& Jones 2001; Biller et al. 2004; Croton et al. 2006; Birzan et al. 2008). While all galaxies are most likely hosting a central black hole, its activity can actually produce two opposite effects: a "negative" feedback, when gas is ejected by the ignition of a nucleus into the intergalactic medium, thus reducing or even stopping star formation (Silk \& Rees 1998; Granato et al. 2001); or a "positive" feedback, when the compression of the inter-stellar medium from the jets induces star-formation (Klamer et al. 2004). Although, based on the data we have, we cannot claim that the AGN should be held responsible for the observed scatter, we notice that the two objects with evidence of AGN activity in our sample (i.e. radio emission) are also those with a sizable plasma component. A more systematic comparison of the radio and gas properties in isolated galaxies should be made, to better assess the role of AGN feedback, also in light of the observed correlation between the asymmetries in the gas morphology and AGN activity in a sample of well studied objects (Diehl \& Statler 2008), which are however in a variety of environments.

\section{The $L_{x}$ vs. age relation}

An attractive explanation of the large intrinsic scatter in the $L_{\mathrm{X}} / L_{B}$ relation of early-type galaxies could be found if we associate it with their merging histories. A first systematic attempt to correlate the X-ray properties with "age" was discussed by Sansom et al. (2000; see also Mackie \& Fabbiano 1997) who noticed a strong deficiency of hot ISM in "young" galaxies, based on a sample of $69 \mathrm{E}$ and S0 galaxies plus 2 merger remnants in a wide range of fine-structure index $\Sigma$, suggested as an indicator of previous mergers (Schweizer \& Seitzer 1992). Five of the sample galaxies are members of the Virgo Cluster, while all the others are either field objects or in loose groups. A refined discussion of X-ray emission versus age is given by Sansom et al. (2006) where spectroscopic or dynamical ages are used, instead of indirect measures such as fine structure. More isolated 


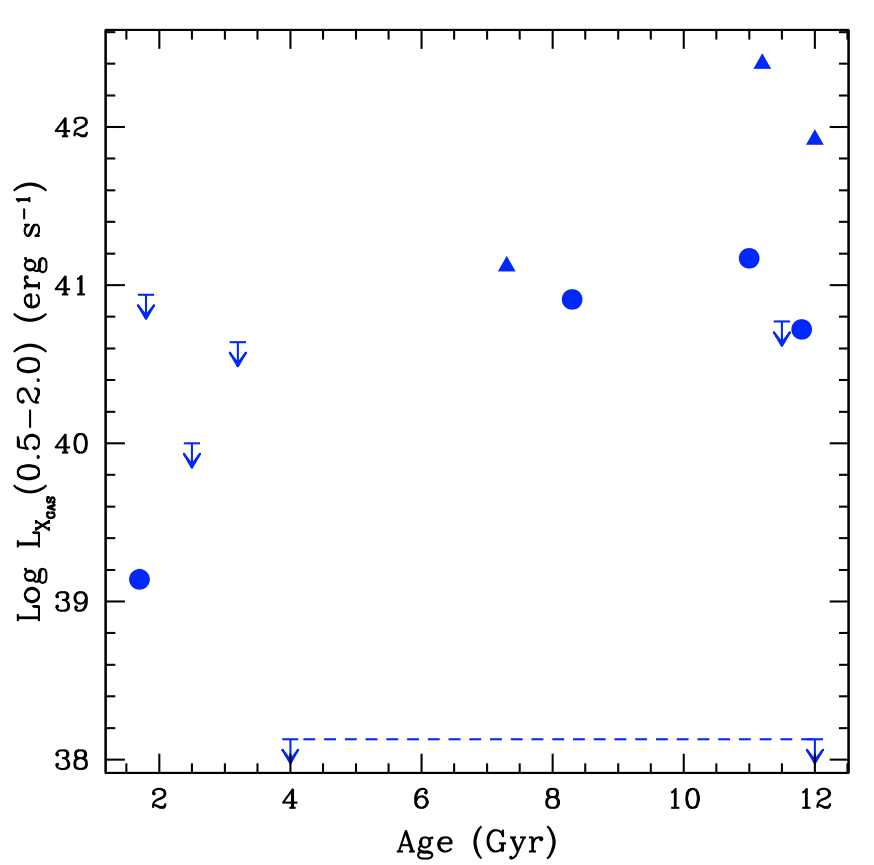

Fig. 9. Scatter plot of the $0.5-2.0 \mathrm{keV}$ hot gas X-ray luminosity vs. age estimates for the isolated galaxies considered in Fig. 8 (see Sect. 6). Fossil groups are plotted as triangles.

examples confirm that merger remnants are under-luminous in $\mathrm{X}$ rays compared with typical mature elliptical galaxies into which these are expected to evolve (Fabbiano \& Schweizer 1995; Nolan et al. 2004). Similarly, in a small sample of pairs located in low density environments, shown in Fig. 8, diffuse hot gas appears to be associated with relaxed objects, while the two systems with unambiguous signatures of ongoing interaction have lower total X-ray emission, indicating a significantly smaller contribution from a hot ISM (Trinchieri \& Rampazzo 2001; Grützbauch et al. 2007).

Due to the paucity of information mainly on ages but also on gas content, most work concentrating on the "age-gas content" relation include all early-type galaxies for which information exists, regardless of the environmental properties that could artificially increase the scatter in the plot, and is not negligible at almost all ages (see Figs. 8 and 9 in Sansom et al. 2006). Here we make an attempt at separating galaxies according to their environment, and compare the ISM properties as a function of age in our sample of isolated galaxies. Unfortunately very few estimates of ages are available. Moreover, it is well known that ages are difficult to interpret properly, since it is often hard to break the age/metallicity degeneracy (see e.g. Trager et al. 1998, 2000; Terlevich \& Forbes 2002), and that ages obtained with different indicators might not be comparable. Therefore, any interpretation of trends with age should be considered carefully.

We found an age of $11 \mathrm{Gyr}$ and $8.3 \mathrm{Gyr}$ for NGC 7052 and NGC 7785, respectively (Terlevich \& Forbes 2002), and $2.5 \pm 0.5$ Gyr for NGC 6172 (Reda et al. 2007). We could find no estimate for the age of NGC 2954. If we assume an age of $\sim 3$ Gyr for this object, which would be consistent with the predicted trend of $B-K$ color with age for a population of solar metallicity (see, e.g. Fig. 5 of Gallagher et al. 2008) ${ }^{2}$, we notice that the two brighter and gas rich galaxies appear to be "old"

\footnotetext{
${ }^{2}$ We are fully aware that this is a rather arbitrary assumption, given the spread of observed $B-K$ color in galaxies at almost any age $>3$ Gyr in the same plot.
}

( $>8 \mathrm{Gyr}$ ), while the low luminosity systems appear to be younger $(<3 \mathrm{Gyr})$.

Again we resort to a search in the literature to enrich the sample, and we add in Fig. 9 the isolated galaxies considered above for which an age estimate is available (NGC 7796: Thomas et al. 2005; NGC 1132, NGC 2271, and NGC 2865: Reda et al. 2007; NGC 6482: Humphrey et al. 2006; NGC 6776, NGC 1600, and ESO107-4: Terlevich \& Forbes 2002; NGC 821: Denicoló et al. 2005 and Proctor et al. 2005). The plot shows a significantly smaller scatter than previous attempts (O'Sullivan et al. 2001a; Samson et al. 2006; Trinchieri et al. 2008a) and seems to indeed suggest that age could be a key factor: young systems, at ages $\leq 3 \mathrm{Gyr}$, are systematically faint (in fact only NGC 2865 is detected). "Old" systems, with ages $>7$ Gyr, appear instead to have a measurable gas component. If we exclude NGC 1132 and NGC 6482 (which are in fact fossil groups, with a significantly larger total mass than our objects), old objects appear to have a gas luminosity of $L_{X} \sim 10^{41} \mathrm{erg} \mathrm{s}^{-1}$. Young shell galaxies, which are typically in a poor environment though not as isolated as the galaxies here, also appear to follow this trend and show a negligible gas content (Trinchieri et al. 2008a).

However, we have a strong case in NGC 821 that could dramatically add to the scatter: its low gas content would suggest a young age (e.g. 4.0 Gyr, Denicoló et al. 2005), however most estimates indicate an older system (7.7 Gyr, Trager et al. 2000; 11.5 Gyr, Caldwell et al. 2003; 12.5 Gyr, Vazdekis et al. 2004). In particular, Pellegrini et al. (2007) adopt an age of $12 \mathrm{Gyr}$ (Proctor et al. 2005), and through hydrodynamical simulations specific for NGC 821 suggest that the hot gas is driven out of the galaxy in a wind sustained by type Ia supernovae, although even with their deep observations they are not able to actually detect it. If the age of NGC 821 is indeed greater than 7 Gyr, than this object alone provides a scatter of about 2 orders of magnitude in the $L_{\mathrm{X}}$ of older objects.

A few attempts at explaining the suggested trend of X-ray luminosity with age have been put forward, and involve both infall of gas or accumulation from the stellar mass loss, after the merging event that has depleted the system from its gas content. Given the extremely poor environment of these systems, infall, either as primordial HI (Hibbard \& van Gorkom 1996) or as hot gas previously being driven out as a result of the merging event, would require a massive dark matter halo to prevent the gas from abandoning the system. O'Sullivan et al. (2001a) argue against infall, which would predict a time-scale for the halo regeneration inconsistent with the observations along the merging sequence, and favour a declining rate in type Ia supernovae that would consequentially reduce the energy deposition in the ISM with time, and produce a transition from outflow (characteristic of younger objects) to inflow and accumulation of the gas shed by stars. Among the old detected objects, NGC 1132 (Mulchaey \& Zabludoff 1999), NGC 6482 (Buote et al. 2007), NGC 1600 (Smith et al. 2008), and NGC 7785 are all relatively massive systems, while NGC 7052 and NGC 7796 (O'Sullivan et al. 2007) appear to be less massive. Except for NGC 1132, the measured gas mass is also consistent with the expected accumulation at the current mass loss rate (see Sect. 5 and Sivakoff et al. 2004; O'Sullivan et al. 2007), which, in this context, would indicate that all systems are massive enough to be able to retain most of the regenerated halo. We have no detailed information on current supernova rate in these systems, to be able to support the O'Sullivan et al. (2007) suggestion of a decline with age. However, we notice that NGC 821 appears to be a significantly less massive system, consistent with it being in an outflow phase (Pellegrini et al. 2007). Interestingly, the observed 
wind in NGC 3379 is also found in an old system with no sizable dark matter halo (see discussion in Trinchieri et al. 2008b). This would point again to mass as one of the key parameters, although, as indicated by NGC 2954 (massive but of unknown age!), mass alone does not seem to be enough to guarantee a sizable hot ISM.

\section{Conclusions}

We have presented the spatial and spectral characteristics of the $\mathrm{X}$-ray emission from four isolated early-type galaxies, selected with strict isolation criteria in the local universe. We find a relatively large scatter in their X-ray properties: two of them have a sizable hot ISM, with $L_{X} \sim 10^{41} \mathrm{erg} \mathrm{s}^{-1}$, while the other two appear to be at least a factor 10 fainter and relatively free of hot gas. A range of X-ray properties was already reported in a few isolated galaxies (e.g. O'Sullivan et al. 2007). In fact, from our systematic attempt to compare the isolated objects available in the literature, we confirm the large scatter in the relation between the X-ray characteristics of their ISM and the stellar luminosity/mass, suggesting that the scatter is not induced by the environment, but it is an intrinsic property of this class of sources. Based on three of our objects, it would also appear that not even the total mass is a discriminant to guarantee the presence of a hot ISM.

Attempts at linking the origin of the scatter with other known galactic properties have not led us to conclusive results. The attractive explanation of relating the ISM content to age appears to be only partially successful: "younger" objects are typically fainter and do not appear to have a sizable hot ISM. Older systems are brighter in their ISM content, however, if we consider the well studied system NGC 821, their X-ray luminosities could span over 2 orders of magnitudes. Understanding the role of the central AGN will require better data than available at the present time.

We are planning to continue our study of the intrinsic properties of early-type galaxies with new X-ray observations and with high quality optical spectra, to obtain a better handle on the age and the metallicity of the stellar populations for a larger set of objects, which in turn will give us insights on possible different evolutions in systems with diverse X-ray properties.

Acknowledgements. We acknowledge financial contribution from the contract ASI-INAF I/023/05/0. We thank the anonymous referee for useful comments and many of our colleagues for interesting discussions during the preparation of this work, in particular: Stefano Andreon, Jay Gallagher, Angela Iovino, and Emanuela Pompei. E.M. is grateful to Lucia Ballo, Lea Giordano, Arturo Mignano, Mara Salvato, Paola Severgnini, Marzia Tajer, and Luca Zappacosta for their scientific support throughout. As one of thousands of Italian researchers with a short-term position, E.M. acknowledges the support of Nature (455, 835-836 and 840-841) and thanks the editors for increasing international awareness of the current critical situation of Italian research. This research made use of SAOImage DS9, and funtools developed by the Smithsonian Astrophysical Observatory (SAO), of the SAO/NASA Astrophysics Data System (ADS), operated by the SAO under a NASA grant, of the NASA/IPAC Extragalactic Database (NED), operated by the Jet Propulsion Laboratory, California Institute of Technology, under contract with the National Aeronautics and Space Administration, and of the Lyon-Meudon Extragalactic DAtabase (LEDA), created in 1983 at Lyon Observatory, and, since that time, continuously updated. Both CIAO and XMM-SAS softwares have been used to reduce the data. This publication made also use of data from the Two Micron All Sky Survey that is a joint project of the University of Massachusetts and the Infrared Processing and Analysis Center/California Institute of Technology, funded by the National Aeronautics and Space Administration and the National Science Foundation. We also used the Digitized Sky Survey that was produced at the Space Telescope Science Institute under US Government grant NAG W-2166, and is based on photographic data of the National Geographic Society - Palomar Observatory Sky Survey (NGS-POSS) obtained using the Oschin Telescope on Palomar Mountain. The NGS-POSS was funded by a grant from the National Geographic
Society to the California Institute of Technology. The plates were processed into the present compressed digital form with their permission.

\section{References}

Andreon, S. 2006, A\&A, 448, 447

Arnaud, K. A. 1996, ASPC, 101, 17

Athey, A., Bregman, J., Bregman, J., Temi, P., \& Sauvage, M. 2002, ApJ, 571, 272

Avni, Y. 1976, 210, 642

Bell, E. F., Mc Intosh, D. H., \& Weinberg, M. D. 2003, ApJS, 149, 289

Bernardi, M., Sheth, R. K., Annis, J., et al. 2003, AJ, 125, 1882

Beuing, J., Dobereiner, S., Bohringer, H., \& Bender, R. 1999, MNRAS, 302, 209

Biller, B. A., Jones, C., Forman, W. R., Kraft, R., \& Ensslin, T. 2004, ApJ, 613, 238

Birzan, L., McNamara, B. R., Nulsen, P. E. J., Carilli, C. L., \& Wise, M. W. 2008, ApJ, 686, 859

Bogdán, Á., \& Gilfanov, M. 2008, MNRAS, 388, 56

Brassington, N. J., Ponman, T. J., \& Read, A. M. 2007, MNRAS, 377, 1439

Brassington, N. J., Fabbiano, G., Kim, D.-W., et al. 2008, ApJS, 179, 142

Brown, B. A., \& Bregman, J. N. 1998, ApJ, 495, L75

Buote, D. A., Gastaldello, F., Humphrey, P. J., Zappacosta, L., et al. 2007, ApJ, 664, 123

Caccianiga, A., Severgnini, P., Braito, V., et al. 2004, 416, 901

Caldwell, N., Rose, J. A., \& Concannon, K. D. 2003, AJ, 125, 2891

Canizares, C. R., Stewart, G. C., \& Fabian, A. C. 1983, ApJ, 272, 449

Canizares, C. R., Fabbiano, G., \& Trinchieri, G. 1987, ApJ, 312, 503

Chiosi, C., \& Carraro, G. 2002, MNRAS, 335, 335

Ciotti, L., D'Ercole, A., Pellegrini, S., \& Renzini, A. 1991, ApJ, 376, 380

Colbert, J. W., Mulchaey, J. S., \& Zabludoff, A. I. 2001, AJ, 121, 808

Colless, M. M., \& the 2dFGRS team 2001, MNRAS, 328, 1039

Colless, M. M., \& the 2dFGRS team 2003, yCat., 7226

Condon, J. J., et al. 2002, yCat, 8065

Croton, D. J., Springel, V., White, S. D., et al. 2006, MNRAS, 365, 11

David, L. P., Jones, C., Forman, W., Vargas, I. M., \& Nulsen, P. 2006, ApJ, 653, 207

De Lucia, G., \& Helmi, A. 2008, MNRAS, 391, 14

Denicoló, G., Terlevich, R., Terlevich, R., Forbes, D. A., \& Terlevich, A. 2005, MNRAS, 358, 813

De Propris, R., Conselice, C. J., Liske, J., et al. 2007, ApJ, 666, 212

Dickey, J. M., \& Lockman, F. J. 1990, ARA\&A, 28, 215

Diehl, S., \& Statler, T. 2008, ApJ, 680, 897

Donato, D., Sambruna, M. N., \& Gliozzi, M. 2004, ApJ, 617, 915

Dressler, A. 1980, ApJ, 236, 351

Eggen, O. J., Lynden-Bell, D., \& Sandage, A. R. 1962, ApJ, 136, 748

Ellis, R. S., Smail, I., Dressler, A., et al. 1997, ApJ, 483, 582

Eskridge, P. B., Fabbiano, G., \& Kim, D. W. 1995, ApJS, 97, 141

Fabbiano, G., \& Trinchieri, G. 1985, ApJ, 296, 430

Fabbiano, G., \& Schweizer, F. 1995, ApJ, 447, 572

Fabricant, D., Rybicki, G. B., \& Gorestein, P. 1984, ApJ, 286, 186

Falco, E. E., Kurtz, M. J., Geller, M. J., et al. 1999, PASP, 111, 438

Ferrarese, L., \& Merritt, D. 2000, ApJ, 539, L9

Finoguenov, A., \& Jones, C. 2001, ApJ, 547, L107

Focardi, P., \& Kelm, B. 2002, A\&A, 391, 35

Focardi, P., \& Kelm, B. 2009, in preparation

Focardi, P., Zitelli, V., Marinoni, S., \& Kelm, B. 2006, A\&A, 456, 467

Forman, W., Jones, C., \& Tucker, W. 1985, ApJ, 293, 102

Fukazawa, Y., Botoya-Nonesa, J. G., Pu, J., Ohto, A., \& Kawano, N. 2006, ApJ, 636, 698

Gallagher, J. S., Garnavich, P. M., Caldwell, N., et al. 2008, ApJ, 685, 752

Granato, G. L., Silva, L., Monaco, P., et al. 2001, MNRAS, 324, 757

Grützbauch, R., Trinchieri, G., Rampazzo, R., et al. 2007, AJ, 133, 220

Gunn, J. E., \& Gott, J. R. III 1972, ApJ, 176, 1

Heckman, T. M. 1983, ApJ, 273, 505

Helsdon, S. F., Ponman, T. J., O'Sullivan, E., \& Forbes, D. A. 2001, MNRAS, 325,693

Hibbard, J. E., \& van Gorkom, J. H. 1996, AJ, 111, 655

Humphrey, P. J., Buote, D. A., Gastaldello, F., et al. 2006, ApJ, 646, 899

Kelm, B., \& Focardi, P. 2004, A\&A, 418, 937

Kelm, B., Focardi, P., \& Sorrentino, G. 2005, A\&A, 442, 117

Kim, D. W., \& Fabbiano, G. 2004, ApJ, 611, 846

Kim, D. W., Fabbiano, G., \& Trinchieri, G. 1992, ApJ, 393, 134

Klamer, I. J., Ekers, R. D., Sadler, E. M., \& Hunstead, R. W. 2004, ApJ, 612, L97

Knochfar, S., \& Silk, J. 2006, ApJ, 648, L21

Larson, R. B. 1975, MNRAS, 173, 671 
Lauer, T. R., Faber, S. M., Richstone, D., et al. 2007, ApJ, 662, 808

Machacek, M., Jones, C., Forman, W. R., \& Nulsen, P. 2006, ApJ, 644, 155

Mackie, G., \& Fabbiano, G. 1997, ASPC, 116, 401

Marconi, A., \& Hunt, L. K. 2003, ApJ, 589, L21

Marcum, P. M., Aars, C. E., \& Fanelli, M. N. 2004, AJ, 127, 3213

Mathews, W. G., Brighenti, F., Faltenbacher, A., et al. 2006, ApJ, 652, L17

Melnick, J., \& Sargent, W. L. W. 1977, ApJ, 215, 401

Mulchaey, J. S., \& Zabludoff, A. I. 1999, ApJ, 514, 133

Nandra, K., \& Pounds, K. A. 1994, MNRAS, 268, 405

Nolan, L. A., Ponman, T. J., Read, A. M., \& Schweizer, F. 2004, MNRAS, 353, 221

Nulsen 1982, MNRAS, 198, 1007

O'Sullivan, E., \& Ponman, T. J. 2004, MNRAS, 349, 535

O'Sullivan, E., Forbes, D. A., \& Ponman, T. J. 2001a, MNRAS, 324, 420

O'Sullivan, E., Forbes, D. A., \& Ponman, T. J. 2001b, MNRAS, 328, 461

O'Sullivan, E., Sanderson, A. J. R., \& Ponman, T. J. 2007, MNRAS, 380, 1409

Pellegrini, S., \& Ciotti, L. 1998, A\&A, 333, 433

Pellegrini, S., Baldi, A., Kim, D. W., et al. 2007a, ApJ, 667, 731

Piconcelli, E., Guainazzi, M., Cappi, M., Jimenez-Bailon, E., \& Schartel, N. 2005, A\&A, 432, 15

Proctor, R. N., Forbes, D. A., Forestell, A., \& Gebhardt, K. 2005, MNRAS, 362, 857

Reda, F. M., Forbes, D. A., Beasley, M. A., O’Sullivan, E. J., \& Goudfrooij, P. 2004, MNRAS, 354, 851

Reda, F. M., Proctor, R. N., Forbes, D. A., Hau, G. K. T., \& Larsen, S. S. 2007, MNRAS, 377, 1772

Revnivtsev, M., Sazonov, S., Gilfanov, M., Churazov, E., \& Sunyaev, R. 2006, A\&A, 452, 169

Revnivtsev, M., Churazov, E., Sazonov, S., Forman, W., \& Jones, C. 2007, A\&A, 473, 783

Revnivtsev, M., Churazov, E., Sazonov, S., Forman, W., \& Jones, C. 2008, A\&A, 490, 37

Sansom, A. E., Hibbard, J. E., \& Schweizer, F. 2000, AJ, 120, 1946
Sansom, A. E., O’Sullivan, E., Forbes, D. A., Proctor, R. N., \& Davis, D. S. 2006, MNRAS, 370, 1541

Sazonov, S., Revnivtsev, M., Gilfanov, M., Churazov, E., \& Sunyaev, R. 2006, A\&A, 450, 117

Schlegel, D. J., Finkbeiner, D. P., \& Davis, M. 1998, ApJ, 500, 525

Schweizer, F., \& Seitzer, P. 1992, IAUS, 149, 488

Silk, J., \& Rees, M. J. 1998, A\&A, 331, L1

Sivakoff, G. R., Sarazin, C. L., \& Carlin, J. L. 2004, ApJ, 617, 262

Smith, R. M., Martínez, V. J., \& Graham, M. J. 2004, ApJ, 617, 1017

Smith, R. M., Martínez, V. J., Fernández-Soto, A., Ballesteros, F. J., \& Ortiz-Gil, A. 2008, ApJ, 679, 420

Stocke, J. T., Keeney, B. A., Lewis, A. D., Epps, H. W., \& Schild, R. E. 2004, AJ, 127, 1336

Sulentic, J. W., Verdes-Montenegro, L., Bergond, G., et al. 2006, A\&A, 449, 937

Terlevich, A. I., \& Forbes, D. A. 2002, MNRAS, 330, 547

Thomas, D., Maraston, C., Bender, R., \& Mendes de Oliveira, C. 2005, ApJ, 621, 673

Tonry, J. L., \& Davis, M. 1981, ApJ, 246, 666

Toomre, A., \& Toomre, J. 1972, ApJ, 187, 623

Trager, S. C., Worthey, G., Faber, S. M., Burstein, D., \& González, J. J. 1998, ApJS, 116, 1

Trager, S. C., Faber, S. M., Worthey, G., \& González, J. J. 2000, AJ, 119, 1645

Treu, T., Stiavelli, M., Casertano, S., Moller, P., \& Bertin, G. 2002, ApJ, 564, L13

Trinchieri, G., \& Fabbiano, G. 1985, ApJ, 296, 447

Trinchieri, G., \& Rampazzo, R. 2001, A\&A, 374, 454

Trinchieri, G., Rampazzo, R., Chiosi, C., et al. 2008a, A\&A, 489, 85

Trinchieri, G., Pellegrini, S., Fabbiano, G., et al. 2008b, ApJ, 688, 1000

Tully, R. B. 1987, ApJ, 321, 280

van der Marel, R. P., \& van den Bosch, F. C. 1998, AJ, 116, 2220

van Dokkum, P. G. 2005, ApJ, 130, 2647

Vazdekis, A., Trujillo, I., \& Yamada, Y. 2004, ApJ, 601, L33

Verdes-Montenegro, L., Sulentic, J., Lisenfeld, U., et al. 2005, A\&A, 436, 443 